\title{
Decreased complications due to application of aerocraft silicone implant in rhinoplasty of preselected patients
}

\author{
Montri Khammoonta, MD', Kyoungjin Kang, MD, PhD ${ }^{2}$ \\ 'Director of Pruksa Medical Clinic, Nakon Pathom, Thailand, ${ }^{2}$ Seoul Cosmetic Surgery Clinic, Seoul, Rep. of Korea
}

\begin{abstract}
Background: In Asia, augmentation rhinoplasty is very common. Although various surgical methods and materials have recently been developed, rhinoplasty using silicone implant remains the most common procedure. However, no standards for the shape and size of the silicone implants exist, and many patients suffer from complications such as deviation, perforation, skin thinning, and extrusion. Objective: We analyzed the effect of performing augmentation rhinoplasty using implant design that craved into the resembled shape of "aerocraft" on nose proportion and complication rates.

Methods: From January 2014 to December 2016, 310 patients who qualified for silicone rhinoplasty based on the indication, considering five parts of silicone corresponding to the nasal anatomy (columella, tip, supratip, dorsum and nasion) underwent augmentation rhinoplasty using the "aerocraft silicone implant." The results of the surgeries and the rates of complications were studied. We performed retrospective analysis of preoperative and postoperative photographs of randomly chosen 42 patients and compared their nose proportion profile.

Results: The results of the modified nasofacial angle showed statistically significant change, but changes in the nasofrontal angle, nasolabial angle, nasomental angle, and nasal tip ratio were not statistically significant. Most of the patients for whom "aerocraft silicone" was used for the rhinoplasty showed very satisfactory aesthetic results during a follow-up of 1 month to 18 months and had only minor complications; the implant removal rate was very low as well.

Conclusion: The use of "aerocraft silicone" for nasal augmentation is safe and effective when performed in selective cases. Good results will be accomplished when the implant is crafted to fit into the patient's nasal anatomy and limits the augmentation to avoid long-term complications.
\end{abstract}

Keywords: aerocraft silicone; Asian rhinoplasty; augmentation rhinoplasty; crafted silicone; silicone rhinoplasty

\section{Introduction}

Most of the Asian population has nose shapes that are different from those of Caucasians, particularly at the nose tip, which is the weakest point, with more fibrotic tissue and thickness. Hence, alterations to the tip point is the most aesthetically demanded change among Asian patients who seek augmentation rhinoplasty [1,2]. However, to enhance the nose tip with a silicone implant, the most popular material in the Asian market, is harmful if it is not limited and performed in selected cases.

We first selected patients for silicone rhinoplasty based on the five criteria as following in materials and methods, subsequently measured the surface anatomy of the nose, and finally crafted the "aerocraft silicone implant" to fit into the patients'

Received August 9 2017, Revised November 10 2017, Accepted November 122017

Corresponding authors: Montri Khammoonta, Pruksa Medical Clinic, No.67/3. Ratcha-Damneon Rd., Phrapathom-Chedi, Muang, Nakon-Pathom 73000, Thailand Tel: 66-3-241-655, E-mail: mt_clinic@hotmail.com

Kyoungjin Kang, Soeul Cosmetic Clinic, 4, Gudeok-ro 34 Beon-gil, Jung-gu, Busan 48594, Rep. of Korea

Tel: 82-51-247-7776, E-mail: kccs7777@gmail.com

This is an Open Access article distributed under the terms of the Creative Commons Attribution Non-Commercial License (http://creativecommons.org/licenses/by-nc/4.0), which permits unrestricted non-commercial use, distribution, and reproduction in any medium, provided the original work is properly cited.

Copyright @ 2017. Korean Society of Korean Cosmetic Surgery (KSKCS) Korean College of Cosmetic Surgery (KCCS). 
nasal anatomy and minimize tension at the nasal tip in order to prevent complications and achieve a satisfactory aesthetic result.

\section{Materials and methods}

We retrospectively reviewed 310 patients that underwent augmentation rhinoplasty using the "aerocraft silicone implant" from January 2014 to December 2016 in my clinic. All the patients were new cases and were operated on using the same techniques. We divided the methods into three steps.

First, we selected qualified candidates for the silicone rhinoplasty. To exclude unqualified patients, the five parameters in Table 1 were considered.

Second, we measured the volume or size (length and thickness) of the silicone implant. To determine the length, the starting point or root of the silicone should begin from the upper two-thirds of the vertical level, from the horizontal line of the medial eye brow and the horizontal line of the medial canthus, and end at the infra-tip lobule (Fig. 1). To estimate the thickness of the nasal dorsum, we used a model of an ideal nose as a template. In the lateral position, a photo of the patient was taken with the patient holding the nasal template. We subsequently viewed the space between the original nose and the new nasal

Table 1. The exclude indication for poor candidate of silicone rhinoplasty

Exclude indication for poor candidate of silicone rhinoplasty

1. Upturn nose and nasolabial angle more than 100 degree

2. Lack of infra tip lobule or hanging alar

3. Short nasal columella height or less than $1 \mathrm{~cm}$.

4. Thick skin and bulbous nasal tip

5. Relative case such as scar contrcture, filler injection, thin skin, infection and perforation of nasal tip

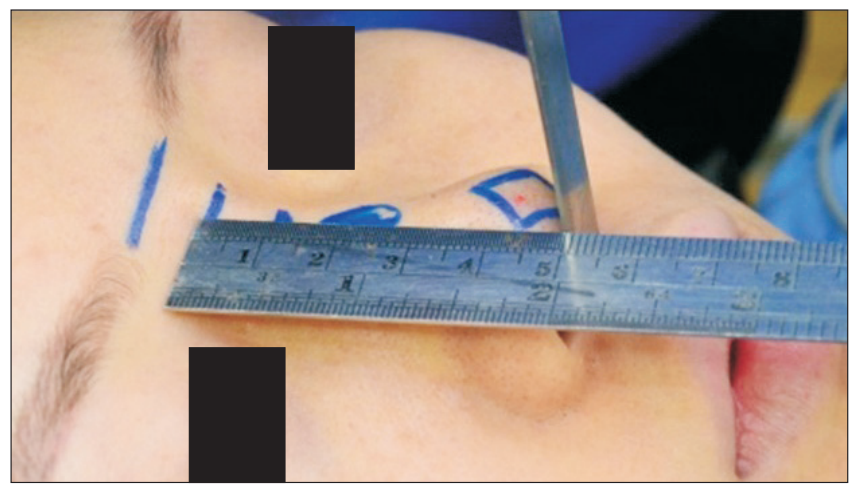

Fig. 1. Measure the length measurement of the silicone implant. shape from the template, which reflects the thickness of the dorsal silicone implant (Fig. 2).

Thereafter, we assessed the volume of the implant to augment the tip using the columellar skin stretch test. The nasal tip was lifted from the base using the digits or a ball-end double hook until the patient can comfortably tightly shut his or her mouth as shown in Fig. 3.

Third, we performed surgery using the carved silicone implant. We used a 35 durometer, 1.3-mm-thick silicone block and a No. 11 blade to carved the silicone. The figure of the "aerocraft silicone implant" was framed to fit each part of the nasal anatomy as shown in Fig. 4.

We performed rhinoplasty using aerocraft silicone implant as a closed technique. An incision was made on both marginal sides to connect through the nasal dome (Fig. 5). A pocket, a bit larger than the width of the silicone implant, was created (Fig. 5). An important aspect was that the undersurface of silicone implant was crafted to cover the small hump at the rhinion, and the silicone's pressure at the nasal tip was estimated by checking the vascular perfusion at the tip (no pallor) and the labial skin should not tend to lift upwards. Thereafter, the implant was inserted without irrigation and its alignment was ascertained before the suture was closed.

We retrospectively reviewed and analyzed, by consecutive sampling, the preoperative and postoperative photographs of 41 women, mean age, 30.86 (18-47) years and one 28-yearold man, who presented at my clinic. All the patents were photographed with an APS-C Digital SLR camera with a Nikon

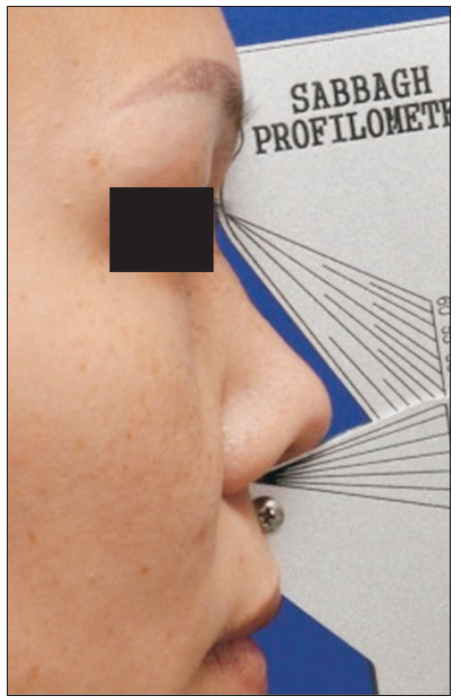

Fig. 2. Measure the thickness of silicone implant by using the ideal nasal template. 

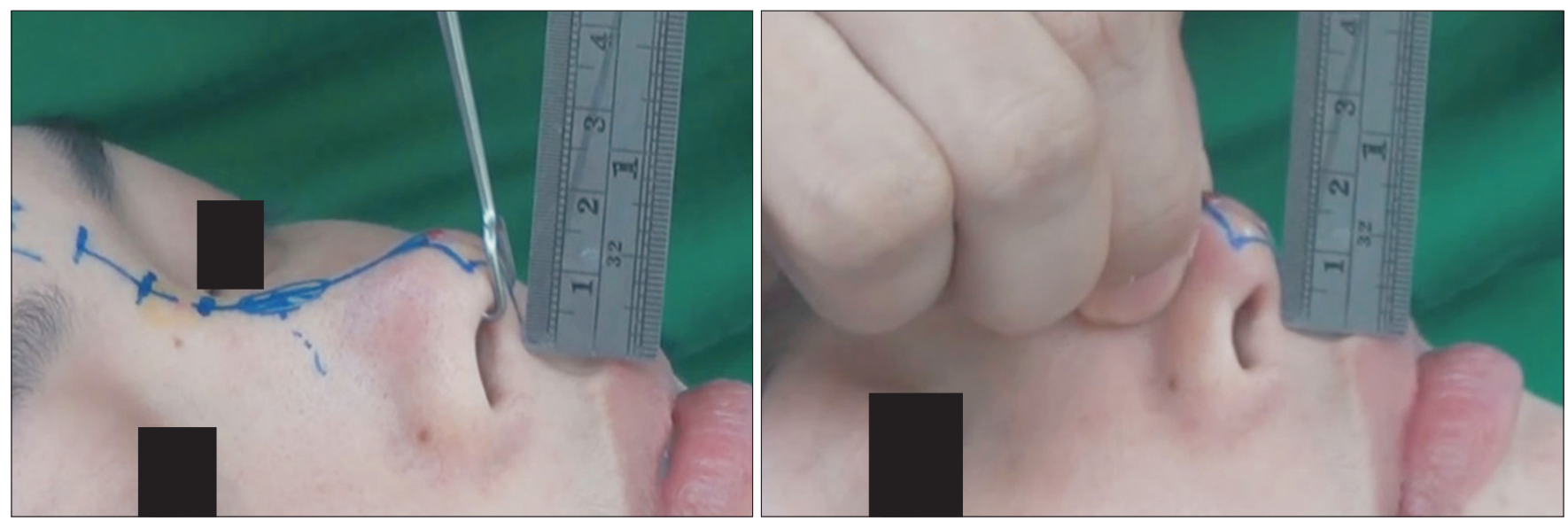

Fig. 3. The guideline for estimate the nasal tip augmentation. The columella skin stretch test by using ball end double hook (left) and the digits to lift up the nasal tip (right).
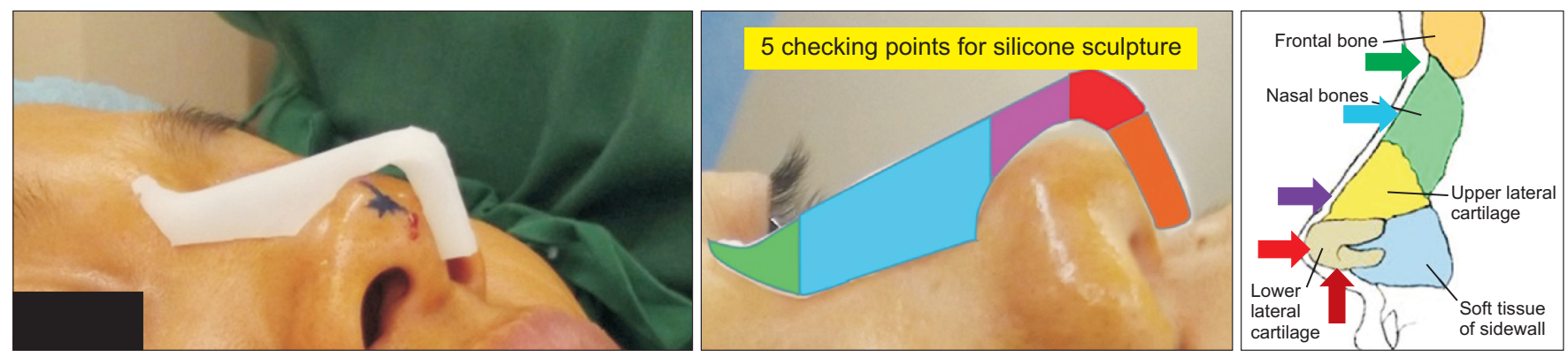

Fig. 4. The "Aerocraft silicone" by manual crafting silicone (left). The drawing picture show different parts of "Aerocraft silicone" (middle). The counterpart of the implant fit to each part of nasal skeleton (right).
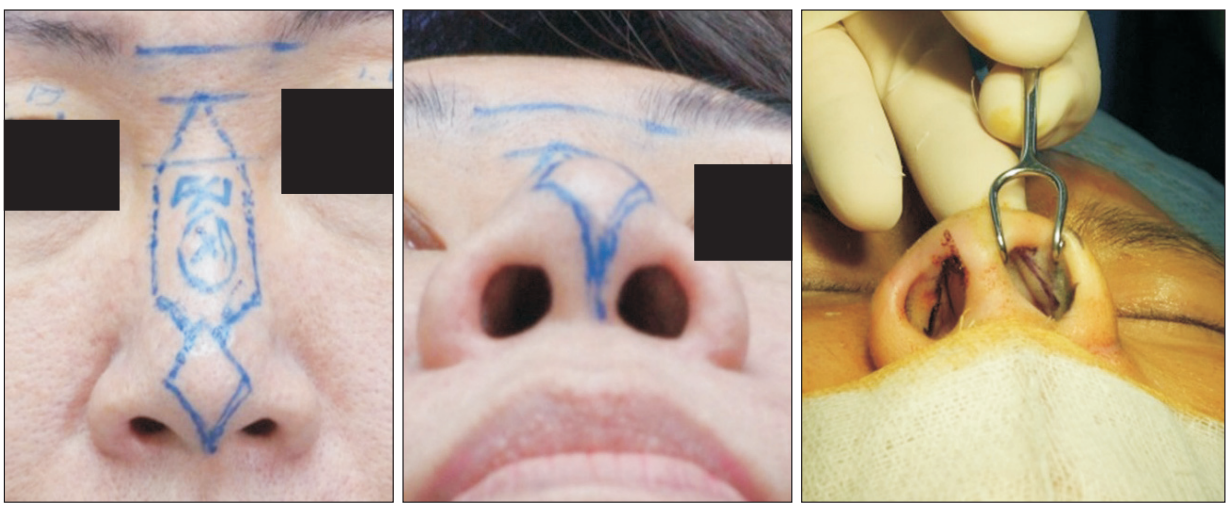

Fig. 5. The nasal skin marking for the "Aerocraft silicone" in frontal view (left), basal view (middle) and both side of the marginal skin incision (right).

lens $35 \mathrm{~mm}$, opened an aperture of $9 \mathrm{~mm}$ and shutter speed of $1 / 60$ second with TTL flash. The pictures were taken before the surgery and during the post-surgical 1 month to 18 months (average, 6 months). We compared the lateral views of the preoperative and postoperative photographs and measured the nasofrontal angle (NFA), modified nasofacial angle (MoNFA), nasolabial angle (NLA), nasomental angle (NMA), and the ratio of the nasal height, as shown in Fig. 6 . The comparison of the 2 groups of photographs was analyzed using the t-test with two paired samples for mean, and $\mathrm{p}<0.05$ was considered statistically significant.

\section{Results}

We examined the nasal profiles of 42 patients (Table 2) and found that the MoNFA of their postoperative lateral view sig- 
nificantly changed $(\mathrm{p}<0.05)$ to a shallow angle because the nasal dorsum had become longer and more projected. The postoperative mean NFA increased but the difference was not statistically significant (mean NFA: $138.63^{\circ}$ preoperatively and $145.6^{\circ}$ postoperatively, $\mathrm{p}>0.05$ ) because the radix was higher and the dorsal part of the nose was longer. However, the mean NLA was slightly changed but no statistically significant difference was found (mean: $95.12^{\circ}$ preoperatively and $95.14^{\circ}$ postoperatively, $\mathrm{p}>0.05$ ). The mean NMA was slightly changed but

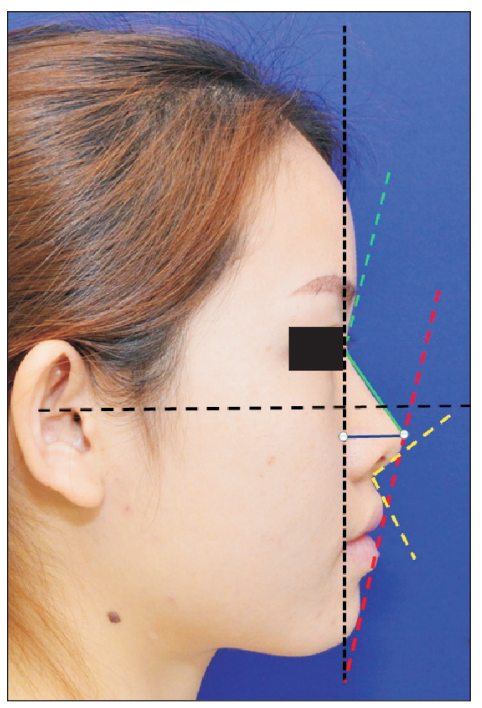

Fig. 6. Measurement of 1. Nasofrontal angle (NFA), The nasofrontal angle was measured at radix as the obtuse angle between a line tangent to the glabella (green dot line) and a line tangent to nasal dorsum line (green dash line). 2. Modified Nasofacial angle (MoNFA), The modified Nasofacial angle was measured at radix as the obtuse angle between a line perpendicular (vertical black dashline) to the Frankfurt horizontal line (horizontal black dash line) and a nasal dorsum line (green solid line). 3. Nasolabial angle (NLA),The nasolabial angle was measured at subnasale as an angle formed between the plane of the columella and upper lip (yellow dash line). 4. Nasomental angle (NMA), The nasomental angle was measured at nasal tip as an angle formed between the nasal dorsum line (green solid line) and the pogonion line (red dash line) and 5. Nasal height raitio, Nasal height ratio was measured perpendicularly at the tip-defining point to the modify facial plane (black solid line) and length of the nasal dorsum from radix to tip-defining point (green solid line). no statistically significant difference was found (mean: $131.98^{\circ}$ preoperatively and $132.55^{\circ}$ postoperatively, $\mathrm{p}>0.05$ ) because the aerocraft implant did not extensively enhance the tip. The mean of the nasal tip height ratio was slightly changed; however, no statistically significant difference was found (mean: $0.66^{\circ}$ preoperatively and $0.56^{\circ}$ postoperatively, $\mathrm{p}>0.05$ ) for the same reason, because the nasal length had increased while the height of the nasal tip was unchanged. Considering the values of the standard deviation (SD) of each nasal profile, the SD had lesser deviation in the most post-operative's profile except the SD of NLA was increase. On the other hand, the aerocraft silicone implant for nasal augmentation improved the each nasal profiles to be better proportion such as NFA, MoNFA, NMA and Nasal tip height ratio. Excepted the NLA was not able to interpret by SD because the value of SD was greater than Mean. Also the value of SD in pre and post operation were not different. That mean the aerocraft silicone implant did not change of NLA (Fig. 7).

A total of 310 cases of aerocraft silicone rhinoplasty were followed up for 1 month to 18 months. The percentage of patients who developed complications was $8 \%, 2 \%$ of whom required implant removal because of infection. The remaining $6 \%$ had minor complications such as skin thinning at the nasal tip, a minor degree of deviation, low nasal bridge, and undercorrection of the nasal tip (Table 3). Evidently, serious complications such as perforations were not encountered, which can be attributed to the cautious selection before the surgery.

\section{Discussion}

Currently, many alloplastic materials for rhinoplasty including silicone, polytetrafluoroethylene, Medpor, AlloDerm, and Proplast are available [3]. However, silicone is the most commonly used because it is biocompatible, cheap, and easy to handle in cases reoperation and removal. Although several authors have reported the successful use of silicone implants such as L-shaped, S-shaped, and bird-shaped silicone or polytetrafluoroethylene and Medpor [4-11] for nose tip enhancement.

Table 2. Profile analysis of nasal augmentation with aerocraft silicone implants

\begin{tabular}{|c|c|c|c|c|c|c|c|c|c|c|}
\hline \multirow{2}{*}{ Analysis } & \multicolumn{2}{|c|}{ Nasofrontal angle } & \multicolumn{2}{|c|}{ Modified nasofacial angle } & \multicolumn{2}{|c|}{ Nasolabial angle } & \multicolumn{2}{|c|}{ Nasometal angle } & \multicolumn{2}{|c|}{ Nasal tip ratio } \\
\hline & Before & After & Before & After & Before & After & Before & After & Before & After \\
\hline $\mathrm{N}$ & 42 & 42 & 42 & 42 & 42 & 42 & 42 & 42 & 42 & 42 \\
\hline Mean & 138.63 & 145.6 & 32.45 & 30.91 & 95.12 & 95.14 & 131.98 & 132.55 & 0.66 & 0.56 \\
\hline SD & 65.9 & 37.13 & 20.74 & 12.77 & 106.4 & 111.83 & 101.54 & 82.11 & 0.42 & 0.01 \\
\hline $\mathrm{p}$ value & \multicolumn{2}{|c|}{$>0.05$} & \multicolumn{2}{|c|}{$<0.05$} & \multicolumn{2}{|c|}{$>0.05$} & \multicolumn{2}{|c|}{$>0.05$} & \multicolumn{2}{|c|}{$>0.05$} \\
\hline
\end{tabular}



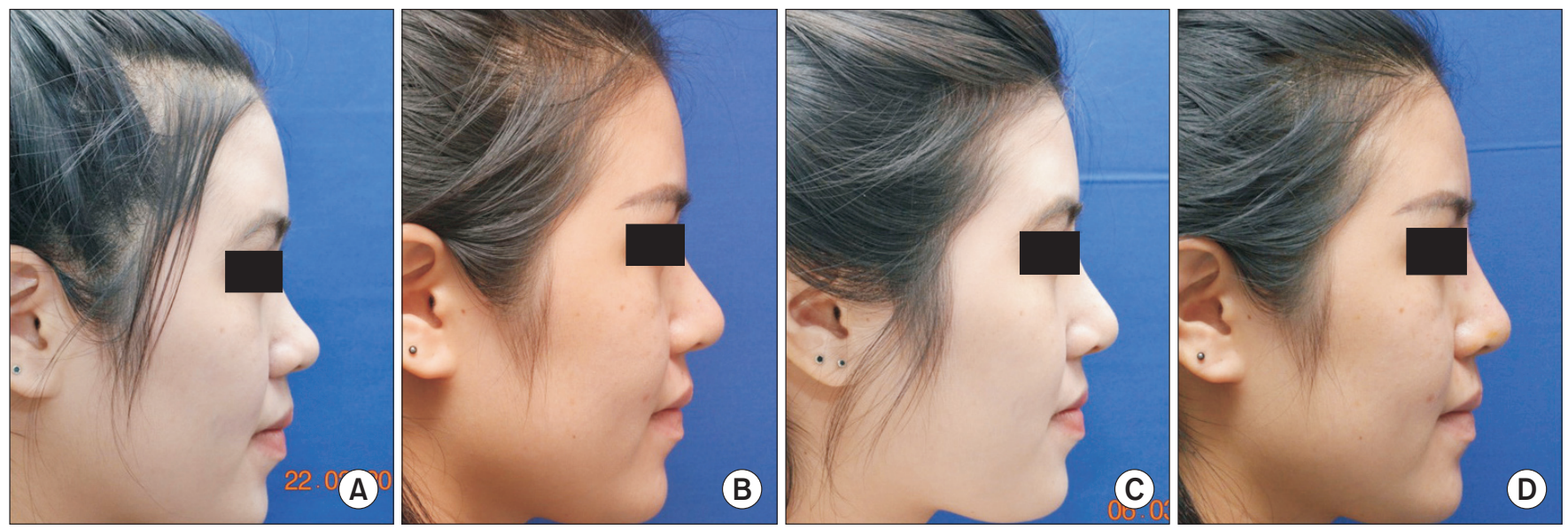

Fig. 7. A case of follow up in 25 -year- old female patient who had performed arhinoplasty using "Aerocraft silicone" implant. A.Before; B,C,D. 1,3 and 12 months after.

Table 3. Incidence of complications by nasal augmentation rhinoplasty using "Aerocraft silicone" implant

\begin{tabular}{lccc}
\hline Complication and complaint & Number & Incidence (\%) & Treatment \\
\hline Infection & 6 & 1.93 & Removal of the implant \\
\hline Thin skin & 3 & 0.96 & Revision by combination with fascia \\
\hline Minor deviation & 2 & 0.64 & No further treatment \\
\hline Low nasal bridge & 6 & 0.64 & No further treatment \\
\hline Under correction of tip & 7 & 2.25 & Augmentation using auto graft \\
\hline Perforation & 0 & 0 & - \\
\hline Total & 24 & 7.74 & - \\
\hline
\end{tabular}

Incidence=number of complicated cases 310 as the total numbers of the patients.

Table 4. Comparison among various shape of nasal silicone implant

\begin{tabular}{lccccc}
\hline \multicolumn{1}{c}{ Complication } & $\begin{array}{c}\text { L shape (7) } \\
(\mathbf{n}=\mathbf{3 3 3})\end{array}$ & $\begin{array}{c}\text { Bird shape (8) } \\
(\mathbf{n}=\mathbf{1 0 7 9 )}\end{array}$ & $\begin{array}{c}\text { S shape (9) } \\
(\mathbf{n}=\mathbf{5 4 8})\end{array}$ & $\begin{array}{c}\text { McGhan } \\
\text { Dorsal columella } \\
\text { strut prosthesis (11) } \\
(\mathbf{n}=\mathbf{4 4 2})\end{array}$ & $\begin{array}{c}\text { Rhinoplasty Japanese (12) } \\
(\mathbf{n}=\mathbf{9 5 4})\end{array}$ \\
\hline Perforation and extrusion & $2(0.6 \%)$ & N/A & $4(0.7 \%)$ & $4(0.9 \%)$ & $32(3.35 \%)$ \\
Displacement & $8(2.3 \%)$ & $32(2.96 \%)$ & $27(4.9 \%)$ & $14(3.16 \%)$ & $40(4.19 \%)$ \\
Infection & $10(2.8 \%)$ & $28(2.6 \%)$ & $2(0.3 \%)$ & N/A & N/A \\
Hematoma & N/A & N/A & $3(0.5 \%)$ & $4(0.9 \%)$ & N/A \\
\hline
\end{tabular}

Nevertheless, the complications from alloplastic augmentation are higher if augmentations are over the skin tension [12]. Anyway we preferred to use the "aerocraft" silicone implant to enhance the nasal tip for every cases by following the method for shapings "aerocraft" silicone implant in order to maintain the transition zone between the tip and the dorsal nose. The "aerocraft silicone implant" can enhance the nasal dorsum significantly while maintaining a low profile of enhancement at the nasal tip.

The standards of the ideal nose are as follows: a straight and narrow bridge; a well-defined projecting nasal tip; refined alae with a $90^{\circ}$ to $95^{\circ}$ NLA in men and $95^{\circ}$ to $100^{\circ}$ in women [13]; a $115-130^{\circ} \mathrm{NFA}, 30-40^{\circ} \mathrm{MoNFA}$, and $120-130^{\circ} \mathrm{NMA}$ [14]; and a nasal tip projection ratio (distance from the tip of the facial plane) of approximately 1:0.67. The morphology of the average Asian nose is remarkably different from these standards and has variations of beauty. The "aerocraft" silicone implant basically enhances the nasal dorsum because it is looser and less fibrotic than the nasal tip in Asians. The mean change in NFA was from $138.62^{\circ}$ to $145.6^{\circ}$ and MoNFA significantly changed from a mean of $32.45^{\circ}$ to $30.90^{\circ}$. However, NLA, NMA and the nasal tip projection ratio were less changed because the "aerocraft 
silicone implant" provided less enhancement at the tip. Further, the implant impacted less tension at the tip and the L-shape of the implant minimized the palpable contour irregularities along the supratip region. Moreover, the wide base of the "aerocraft silicone implant" covered the dorsum more laterally and reduced the visible and palpable edges in very thin patients.

Considering the risks of rhinoplasty [10], we compared the complications of "aerocraft silicone implant" with those of implants of various shapes (Table 4). Complications related to "aerocraft silicone implant" such as perforation and deviation have significantly low rate, although another complication (infection) was not significantly different. The preoperative analysis carried out was essential to predict and prevent nasal tip destruction through perforation, which can cause skin deformity and difficulty in correction. The "aerocraft silicone implant" was suitable for augmenting the nasal dorsum and tip, with acceptable complication rate.

\section{Conclusion}

From the above results, it is suggested that augmentation rhinoplasty using "aerocraft silicone implant" with prior nasal analysis is relatively safe in Asians. This is attributable to appropriate patient selection and application of the anatomically and functionally suitable "aerocraft silicone implant."

\section{Conflicts of interest}

The authors have nothing to disclose.

\section{References}

1. Jang YJ. Rhinoplasty and septoplasty. Seoul: Koonja Publisher; 2014. p. 239-61.

2. McCurdy JA Jr. Asian rhinoplasty. In: McCurdy JA Jr, Lam SM, editors. Cosmetic surgery of the Asian face. 2nd ed. New York:
Thieme Medical Publishers; 2005. p. 42-67.

3. Suh MK. Dorsal augmentation with implants. In: Suh MK, editor. Asian rhinoplasty. Seoul: Korea Academy of Plastic Surgery; 2012. p. 71-102.

4. McCurdy JA Jr. The Asian nose: augmentation rhinoplasty with L-shaped silicone implants. Facial Plast Surg 2002;18: 245-52.

5. Park JI. Introduction to augmentation rhinoplasty. In: Park JI, Toriumi DM, editors. Asian facial cosmetic surgery. Philadelphia: Saunders Elsevier; 2007. p. 137-44.

6. Kim IS. Augmentation rhinoplasty using silicone implants. In: Jin HR, editor. Aesthetic plastic surgery of the East Asian face. New York: Thieme Medical Publishers; 2016. p. 13-25.

7. Tham C, Lai YL, Weng CJ, Chen YR. Silicone augmentation rhinoplasty in an Oriental population. Ann Plast Surg 2005;54:1-5.

8. Lam SM, Kim YK. Augmentation rhinoplasty of the Asian nose with the "bird" silicone implant. Ann Plast Surg 2003;51: 249-56.

9. Chuangsuwanich A, Lohsiriwat V. Augmentation rhinoplasty with custom-made S-shape silicone implant in Asians: a 15year experience. Indian J Plast Surg 2013;46:533-7.

10. Rettinger G. Risks and complications in rhinoplasty. GMS Curr Top Otorhinolaryngol Head Neck Surg 2007;6:Doc08.

11. Deva AK, Merten S, Chang L. Silicone in nasal augmentation rhinoplasty: a decade of clinical experience. Plast Reconstr Surg 1998;102:1230-7.

12. Hiraga Y. Complications of augmentation rhinoplasty in the Japanese. Ann Plast Surg 1980;4:495-9.

13. Jang D, Yu L, Wang Y, Cao D, Yu Z, Mu X. Nasal measurements in Asians and high-density porous polyethylene implants in rhinoplasty. Arch Facial Plast Surg 2012;14:181-7.

14. Prendergast PM. Facial proportions. In: Erian A, Shiffman MA, editors. Advanced surgical facial rejuvenation: art and clinical practice. Berlin: Springer; 2012. p. 15-22. 